\title{
Simultaneous CFA Demosaicking of Three Color Planes for Improved Color Accuracy
}

\author{
Omar Shakar, Jim S. Jimmy Li*, Sharmil Randhawa \\ College of Science and Engineering, Flinders University, GPO Box 2100, Adelaide, SA 5001, Australia. \\ * Corresponding author. Tel.: +61 8 82015050; email: jimmy.li@flinders.edu.au \\ Manuscript submitted March 5, 2019; accepted May 5, 2019. \\ doi: $10.17706 /$ jcp.14.5.318-327
}

\begin{abstract}
Color Filter Array (CFA) demosaicking is an interpolation process of reconstructing a full color image from a single image sensor. Conventionally, color planes are demosaicked sequentially. The green color plane is usually demosaicked first due to the fact that it contains twice the information of the other color planes for the Bayer pattern, and then followed by the demosaicking of red and blue color planes. However, this sequential process of demosaicking will produce color artifacts which cannot be recognized until all the color planes have been demosaicked. In our paper, we propose a novel technique to demosaic the three color planes simultaneously so as to avoid the selection of artifact pixels. Based on the interpolation in different directions to estimate a full color pixel for edge preservation, multiple combinations of RGB color values for a pixel location are generated. Among those RGB combinations, some are good estimates while others are color artifacts. Using the color-line property to discriminate those color artifact pixels, an optimum RGB color value from those good estimates will be selected. It has been shown that our proposed algorithm outperforms the other benchmarking algorithms and produces minimal color artifacts. The experimental results have shown consistency in performance in both perceptual and objective evaluation for various types of image datasets.
\end{abstract}

Key words: Simultaneous CFA demosaicking, color artifacts, color-line property, image segmentation.

\section{Introduction}

Most digital cameras capture only a single color per pixel using a single image sensor. This sensor is covered by a color filter array (CFA), such as the most common Bayer [1] pattern as shown in Fig. 1, to obtain one of the three primary colors (red, green, or blue) at each pixel location. An interpolation process called demosaicking is then applied to produce a full color image. Many demosaicking methods have been proposed for reconstructing a full color image including method of residue interpolations [2]-[4], edge directed interpolation [5]-[15], and frequency domain [16], [17]. However, all of them still suffer from some degree of color artifacts due to problems such as cross-channel aliasing and interpolation across edges. Color artifacts often manifest themselves as false color [18]-[22] and zipper effect [6], [21]-[23]. As color artifacts can only be identified when the full color of a pixel is determined, the conventional methods of demosaicking three color planes sequentially will be unable to avoid color artifacts which will only appear after the demosaicking process has completed. In our proposed method, all the three color planes are demosaicked simultaneously so that in the estimation of a missing color, potential estimates which are color artifacts can be identified within the process and avoided. 
In order to discriminate potential estimates which are color artifacts, our proposed method incorporates the color-line property which states that the local RGB color values tend to distribute linearly along a regression line in RGB color space for natural color images [24], [25]. Hence, color artifact pixels are those outliers that deviate from the regression line. To preserve sharp edges, multiple combinations of RGB values obtained by interpolants in different directions are produced, and an optimum color value which is nearest to the corresponding line is then selected. In this way, the optimum color values selected are virtually free from color artifacts.

The remainder of this paper is organized as follows. Section 2 introduces our proposed demosaicking method. Section 3 presents the quantitative and visual assessment comparison results of our proposed method with other benchmarking algorithms. The conclusion is given in Section 4 .

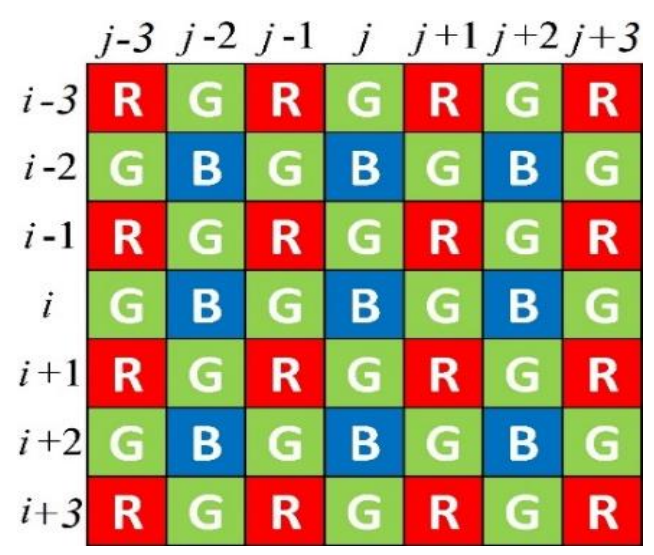

Fig. $1.5 \times 5$ Bayer CFA pattern.

\section{Proposed Demosaicking Algorithm}

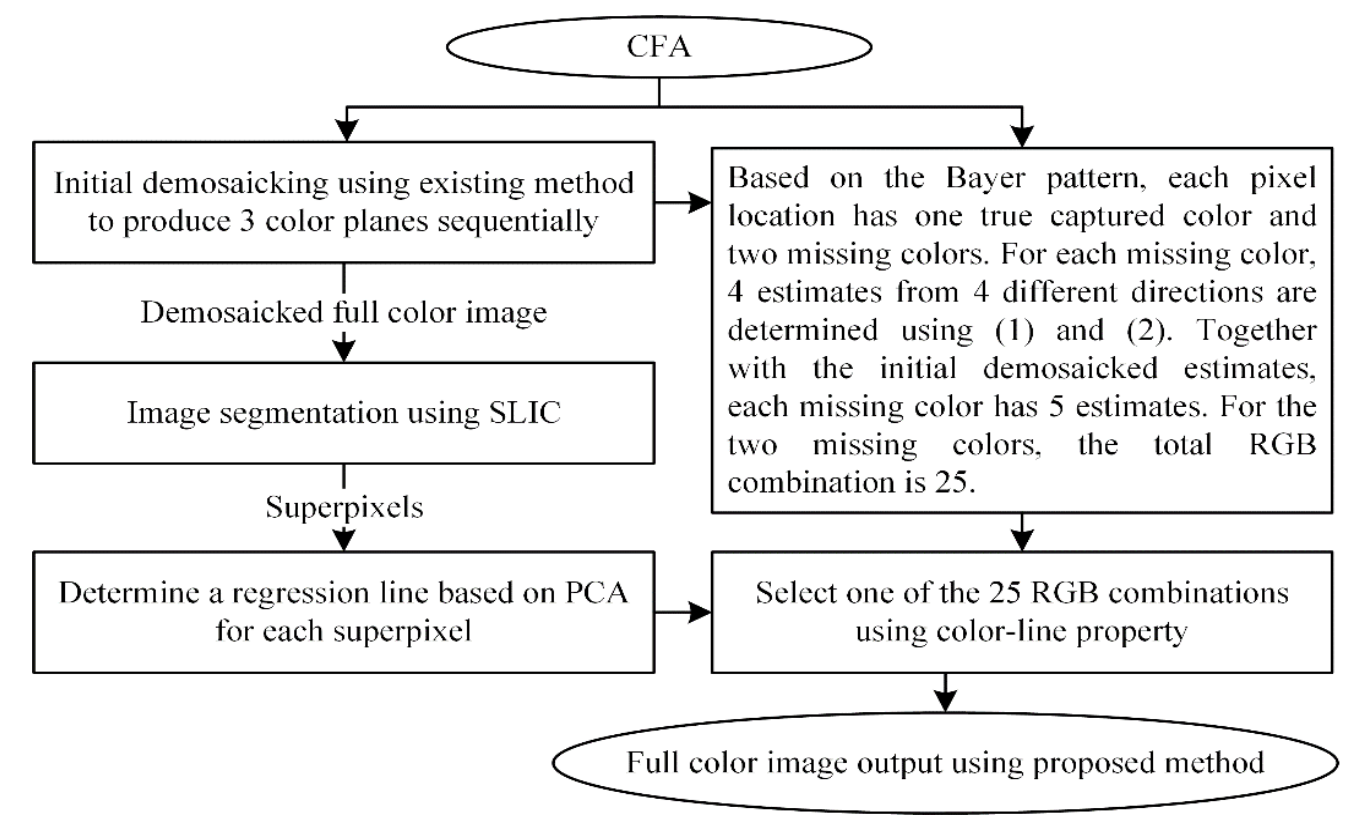

Fig. 2. Flowchart of the proposed demosaicking method.

Fig. 2 gives the flowchart of our proposed method by simultaneous demosaicking of three color planes. Section 2.1 describes the interpolation of the red, green and blue planes. Section 2.2 gives the details on the 
color-line property, and the following Section 2.3 on image partitioning. Section 2.4 gives the details on the orientation of the regression line in color-line property, and Section 2.5 describes the method to select the optimum color pixel from 25 RGB combinations.

\subsection{RGB Combinations of A Color Pixel}

Based on the Bayer pattern as shown in Fig. 1, each color pixel has one true captured color and two missing colors. In this section, we will determine all the possible color combinations of a color pixel. For each combination, the true captured color remains unchanged. For each missing color, four interpolants from four orthogonal directions are determined for edge preservation [11]. As the estimate for a missing color using the initial demosaicking method has good accuracy for smooth region, it should also be included. As a result, together with this estimate and the four interpolants for the one missing color, there will be a total number of $25 \mathrm{RGB}$ combinations for the two missing colors.

To estimate the missing red color value at the blue pixel location $(i, j)$ in Fig. 1 , four estimates from different directions, namely top-left $(T L)$, top-right $(T R)$, bottom-left $(B L)$ and bottom-right $(B R)$, are determined by using first-order interpolation [11]. The red estimate at location $(i, j)$ from the top-left direction, $\hat{R}_{i, j}^{T L}$, is given as follows:

$$
\hat{R}_{i, j}^{T L}=R_{i-1, j-1}+\left(\tilde{G}_{i, j}-\tilde{G}_{i-1, j-1}\right)
$$

where $R_{i-1, j-1}$ is the true red captured value at the location $(i-1, j-1)$, and $\tilde{G}_{i, j}$ and $\tilde{G}_{i-1, j-1}$ are the green estimates by the initial demosaicking method at location $(i, j)$ and $(i-1, j-1)$ respectively. For the estimates for the other three directions $\left(\hat{R}_{i, j}^{T R}, \hat{R}_{i, j}^{B L}\right.$ and $\left.\hat{R}_{i, j}^{B R}\right)$, similar equations can be applied.

For the missing green at the same location $(i, j)$, four directional estimates at top, bottom, left and right directions, namely, $\hat{G}_{i, j}^{T}, \hat{G}_{i, j}^{B}$, $\hat{G}_{i, j}^{L}$ and $\hat{G}_{i, j}^{R}$ are determined respectively using second-order interpolation [11]. The green estimate at location $(i, j)$ from the top direction, $\hat{G}_{i, j}^{T}$, is given as follows:

$$
\hat{G}_{i, j}^{T}=G_{i-1, j}+\frac{1}{2}\left(B_{i, j}-B_{i-2, j}\right)+\frac{1}{8}\left(G_{i+1, j}-2 G_{i-1, j}+G_{i-3, j}\right)
$$

where $G_{i-1, j}$ is the true captured green value at location $(i-1, j)$ and $B_{i, j}$ is the true captured blue color value at location $(i, j)$. The other estimates $\hat{G}_{i, j}^{B}, \hat{G}_{i, j}^{L}$ and $\hat{G}_{i, j}^{R}$ can be determined similarly [11]. At the other color pixel locations in the Bayer pattern, the above equations can be applied similarly.

\subsection{Color-Line Property}

From the previous section, 25 RGB color combinations for a color pixel location have been determined. In this section, we propose using color-line property to select an optimum color value, which will be free from color artifacts, from those 25 different color combinations.

Color-line property is the local distribution of color pixels of a color image in the RGB color space. The color-line property states that natural color images have RGB color pixel values within a local region distributing linearly along a line in the RGB color space [24]. Hence, color artifact pixels can be easily identified as outliers that deviate from the corresponding line.

Fig. 3(a) and (b) shows an example of the color-line properties of a natural image region with and without color artifacts respectively. As shown in Fig. 3(a), the linear relationship of the color pixels distribution is demonstrated in a natural color image by the color-line property. However, in the presence 
of color artifacts in the same local region of the image, the color-line property has a wider dispersion due to the deviation of those color artifact pixels from the center regression line, as shown in Fig. 3(b).

By choosing one of those 25 RGB combinations found in Section 2.1 that is closest to the center of regression line, a color pixel which is free from color artifacts will be selected.
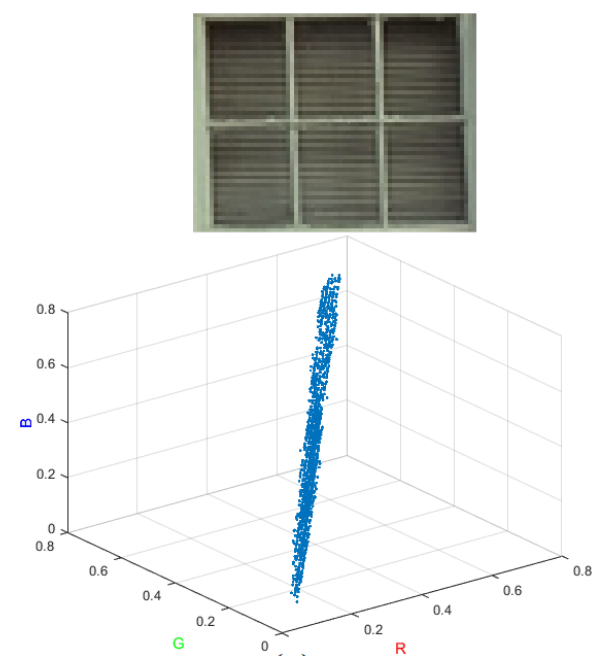

(a)

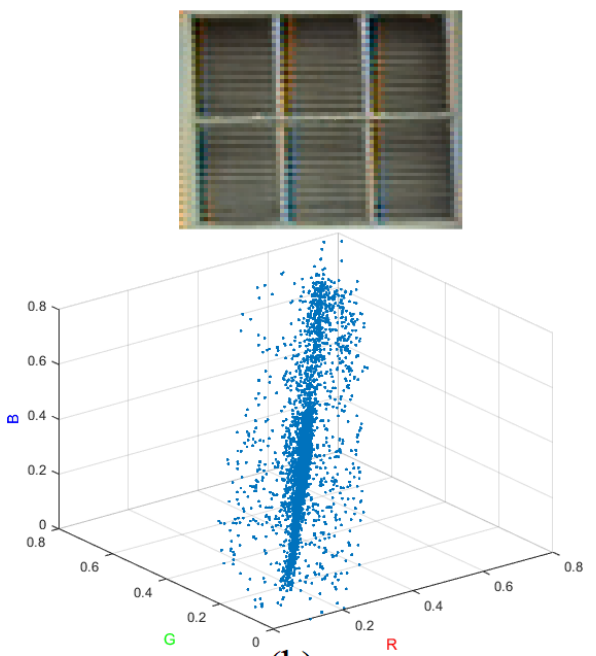

(b)

Fig. 3. Color-line property of local region extracted from image: (a) without color artifacts, and (b) with color artifacts.

\subsection{Image Segmentation}

As the color-line property will only be satisfied within a local region, the image must be segmented prior to the application of the color-line property. The pixels in a local region are required to be similar with respect to some characteristics, such as color homogeneity in order to make the color-line property applicable in each local region.

Since the CFA input image does not contain enough information for image segmentation, it will first be demosaicked to a full color image prior to its segmentation. As minor color artifacts produced by the initial demosaicking technique has minimal effects on the results of segmentation, the WM-HOI demosaicking method in [11] was used for initial demosaicking of the CFA input image. However, other demosaicking methods [4], [10]-[12], [15], [17] can be chosen.

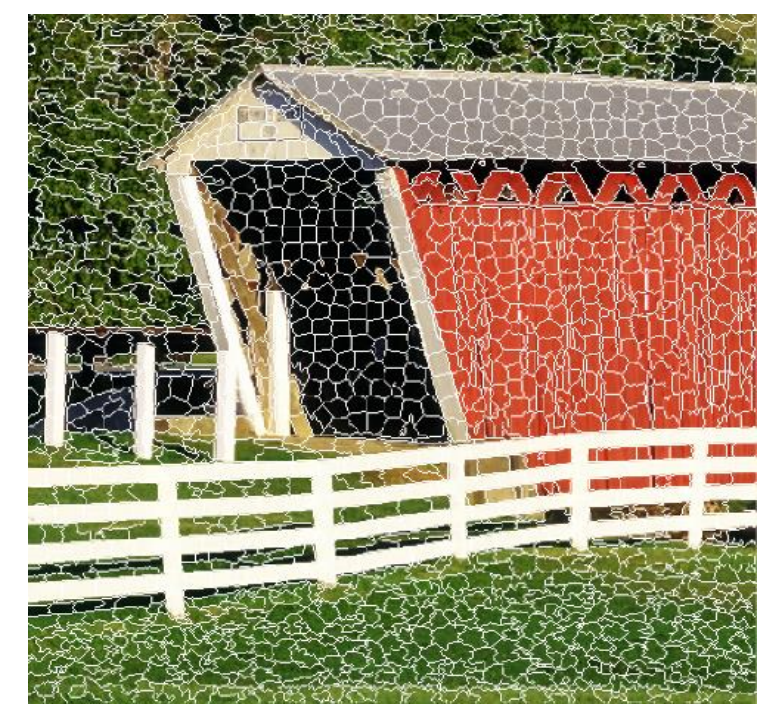

Fig. 4. A segmented image using SLIC superpixel algorithm. 
In order to obtain a well - defined regression line within a local region to satisfy the color-line property, the image must be segmented matching object boundaries. Simple linear iterative clustering (SLIC) superpixel algorithm [26] is well suited for this application as it can generate superpixels as local regions which will better match object boundaries, where a superpixel is a small set of pixels with homogeneous color that perceptually have a meaningful atomic region. Fig. 4 gives the segmented output of an image from IMAX dataset [10] using the SLIC algorithm as an example. As shown in Fig. 4, the image was segmented such that each superpixel consists of similar color pixels within an object boundary.

\subsection{Orientation of the Regression Line in Color-Line Property}

As we require a regression line for each superpixel as a reference to choose an optimum color value from the 25 RGB combinations, the superpixels obtained from the initial demosaicked image are used. As only the orientation of a regression line is required for the selection of one of the 25 RGB combinations, minor color artifacts produced by the initial demosaicking technique has minimal effects on the accuracy of the orientation of the regression line as well.

To approximate the regression line of a superpixel in the segmented image, Principle Component Analysis (PCA) [27] was applied to construct a best fitted regression line which is constructed based on the largest eigenvalue and its corresponding eigenvector. To improve the accuracy of the regression line, half of the color pixels which are furthest away from the regression line are discarded. The regression line is then reconstructed again based on the recalculated largest eigenvalue and its corresponding eigenvector using the remaining color pixels.

\subsection{Optimum Color Pixel Selection from 25 RGB Combinations}

The regression line found in Section 2.4 is used as a reference in the selection of the optimum color pixel. Let $S$ be the superpixel segment, which is decomposed by PCA as follows:

$$
S=U \Lambda U^{T}
$$

where $\Lambda=\operatorname{diag}\left\{\lambda_{1}, \lambda_{2}, \lambda_{3}\right\}$ is the $3 \times 3$ diagonal eigenvalue matrix, $\lambda_{1}, \lambda_{2}$ and $\lambda_{3}$ are the eigenvalues, s.t. $\lambda_{1} \geq \lambda_{2} \geq \lambda_{3}$ and $U^{T}$ is the $3 \times 3$ eigenvector matrix. Let $\vec{u}=\left[\begin{array}{lll}u_{1,1} & u_{1,2} & u_{1,3}\end{array}\right]$ be the first row of $U^{T}$ matrix representing the eigenvector for $\lambda_{1}$, and its corresponding eigenvector, $\vec{u}$, gives the direction of the regression line.

To select the optimum color value from one of the 25 RGB combinations found in Section 2.1, the orthogonal distance from each of the 25 RGB combinations to the regression line is first determined. The optimum color value is the one which has the minimum orthogonal distance to the regression line.

Let $\mathrm{d}_{n}$ be the orthogonal distances between a color pixel to the regression line given by $\vec{u}$, and $D$ be the minimum orthogonal distance value among, $\left\{d_{n}\right\}$, where $n=1,2, \ldots, 25$, given as follows:

$$
D=\min \left(\left\{d_{n}\right\}\right)
$$

where $n=1,2, \ldots, 25$. The color pixel is therefore the optimum color pixel with an orthogonal distance equal to $D$.

\section{Experimental Results}

To assess the performance of our proposed demosaicking method quantitatively and visually, it was compared to six benchmarking demosaicking methods, namely WM-HOI [11], LSLCD [17], LDI-NAT [10], 
MDWI [12], RI [4], and DCD [15]. Two different color image datasets, namely Kodak [28] and IMAX [10] were used as they have a different degree of inter-channel correlation [10], [29], [30]. Kodak images are known to have less saturated appearance with strong inter-channel correlation [10], while IMAX images are deemed to be closer to images captured by image sensors and have low inter-channel correlation [29], [30].

Four different image quality assessment (IQA) methods, namely color peak signal-to-noise ratio (CPSNR) [31], gradient magnitude similarity deviation (GMSD) [32], zipper effect (ZE) [6], and normalized color variation (NCV) [33] were used to quantitatively evaluate the performance of each demosaicking method. Table 1 and Table 2 show the average numerical results for the performances of our proposed demosaicking method against the six benchmarking demosaicking methods using all the test images from the Kodak and IMAX datasets respectively. Our proposed method achieved the best results in all the four IQA measures, and outperformed the six demosaicking methods for the Kodak image dataset as shown in Table 1. For the IMAX image dataset, our proposed method is the second best for CPSNR [31] but remains the best for all the other three IQA measures. As color artifacts are visible errors while CPSNR measures the total errors including both visible and non-visible errors, CPSNR does not correlate as well as the other three IQA measures with visual assessment which was confirmed in our next qualitative visual comparison.

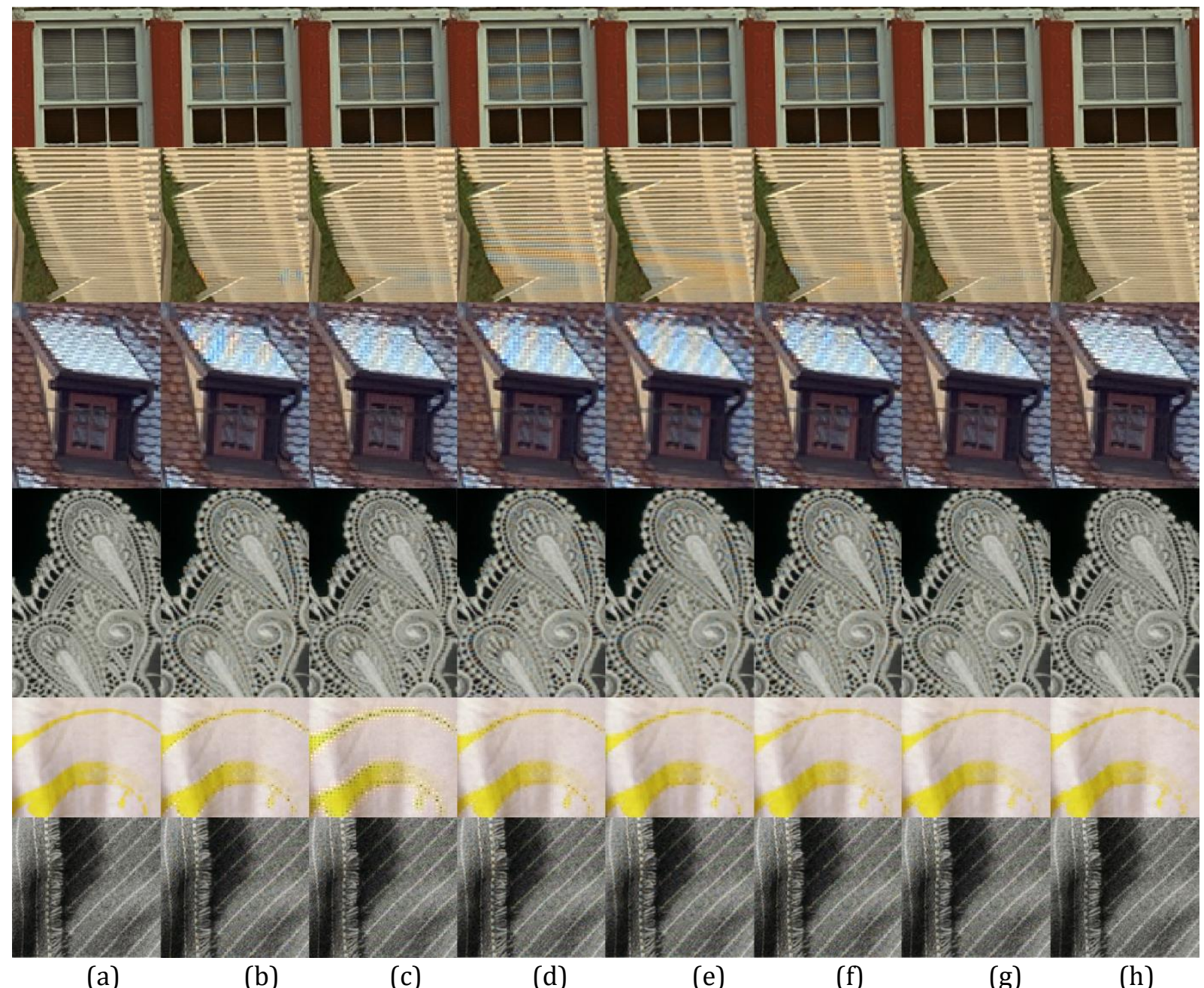

Fig. 5. Cropped regions of (a) the original image, and the demosaicked images using (b) WM-HOI [11], (c) LSLCD [17], (d) LDI-NAT [10], (e) MDWI [12], (f) RI [4], (g) DCD [15], and (h) the proposed algorithm.

The visual comparison of our proposed method with the other benchmarking demosaicking algorithms is 
given in Fig. 5. The first three images of Fig.5 (a) are cropped regions of Kodak images, and the remaining three images are cropped regions of IMAX images. The demosaicked images of Fig. 5 (b)-(g) were produced by the six benchmarking demosaicking algorithms, namely, WM-HOI [11], LSLCD [17], LDI-NAT [10], MDWI [12], RI [4], and DCD [15] respectively, and the images in Fig. 5 (h) were produced by our proposed method. Those cropped regions are known to cause severe color artifacts in most demosaicking algorithms. As shown in the first three images in Fig. 5 (h), our proposed algorithm produced virtually invisible color artifacts in those regions. This agrees with the quantitative results in Table 1 that our proposed method is the best for the Kodak image dataset. As shown in the last three IMAX demosaicked images in Fig. 5 (h), our proposed method also produced negligible color artifacts in those regions. In comparing with the demosaicked images produced by RI [4] as shown in Fig. 5 (f), the demosaicked images produced by our proposed method as shown Fig. 5 (h) contains far less amount of color artifacts, even though the CPNSR result of our proposed demosaicking method is slightly less than that of the RI [4] method.

Table 1. The Average CPSNR [31], GMSD [32], ZE [6] and NCV [33] Values for Kodak Dataset [28] with the Best in Bold

\begin{tabular}{lcccc}
\hline \hline & CPSNR (dB) & GMSD $\left(\times 10^{-2}\right)$ & ZE $(\%)$ & NCV $\left(\times 10^{-3}\right)$ \\
\hline WM-HOI [11] & 39.05 & 1.578 & 7.23 & 0.845 \\
LSLCD [17] & 39.23 & 1.623 & 6.93 & 0.694 \\
LDI-NAT [10] & 37.70 & 2.798 & 8.15 & 1.790 \\
MDWI [12] & 37.04 & 1.680 & 12.06 & 1.606 \\
RI [4] & 38.99 & 1.378 & 7.81 & 0.981 \\
DCD [15] & 39.43 & 1.130 & 6.76 & 0.604 \\
Proposed & $\mathbf{3 9 . 7 1}$ & $\mathbf{1 . 1 2 6}$ & $\mathbf{6 . 5 8}$ & $\mathbf{0 . 5 1 1}$ \\
\hline \hline
\end{tabular}

Table 2. The Average CPSNR [31], GMSD [32], ZE [6] and NCV [33] Values for IMAX Dataset [10] with the

\begin{tabular}{lcccc}
\hline \multicolumn{5}{c}{ Best in Bold } \\
\hline WM-HOI [11] & 35.02 & 2.500 & 12.65 & NCV(x10-3) \\
LSLCD [17] & 32.56 & 4.567 & 18.03 & 0.344 \\
LDI-NAT [10] & 35.57 & 1.766 & 12.32 & 3.337 \\
MDWI [12] & 36.07 & 2.061 & 14.16 & 0.373 \\
RI [4] & $\mathbf{3 6 . 7 2}$ & 1.672 & 12.10 & 0.251 \\
DCD [15] & 35.46 & 2.300 & 12.05 & 0.234 \\
Proposed & 36.13 & $\mathbf{1 . 5 6 7}$ & $\mathbf{1 2 . 0 0}$ & $\mathbf{0 . 1 9 7}$ \\
\hline \hline
\end{tabular}

\section{Conclusion}

By demosaicking of the three color planes simultaneously and using the color-line property, pixels containing color artifacts can be identified and excluded from being selected from those possible RGB combinations within the process to obtain an optimum color value, resulting in a demosaicked image which is free from color artifacts. It has been shown that our novel proposed method outperformed the other benchmarking methods quantitatively and visually in our experimental results. Our future research includes the implementation of our proposed method on dedicated hardware for real-time processing. 


\section{References}

[1] Bayer, B. E. (1976). Color Imaging Array. US Patent 3971065.

[2] Kiku, D., Monno, Y., Tanaka, M., \& Okutomi M. (2013). Residual interpolation for color image demosaicking. Proceedings of IEEE International Conference on Image Processing (ICIP) (pp. 2304-2308).

[3] Kiku, D., Monno, Y., Tanaka, M., \& Okutomi, M. (2014). Minimized laplacian residual interpolation for color image demosaicking. Proceedings of SPIE Electronic Imaging (EI), Digital Photography X, 9023 (pp. 90230L-1-8).

[4] Kiku, D., Monno, Y., Tanaka, M., \& Okutomi M. (2016). Beyond color difference: Residual interpolation for color image demosaicking. IEEE Transactions Image Processing, 25(3), 1288-1300.

[5] Li, J. S. J., \& Randhawa, S. (2005). High order extrapolation using Taylor series for color filter array demosaicing. Lecture Notes in Computer Science Series Berlin: Springer-Verlag, LNCS 3656, 703-711.

[6] Lu, W., \& Tan, Y. (2003). Color filter array demosaicking: New method and performance measures. IEEE Transactions Image Processing, 12(10), 1194-1210.

[7] Li, J. S. J., \& Randhawa, S. (2007). Colour filter array demosaicking using cubic spline interpolation. Proceedings of IEEE International Conference on Acoustics, Speech, and Signal Processing (pp. I865-I868).

[8] Zhang, L., \& Wu, X. (2005). Color demosaicking via directional linear minimum mean square-error estimation. IEEE Transactions Image Processing, 14(12), 1057.

[9] Li, J. S. J., \& Randhawa, S. (2008). Weighted median based colour filter array demosaicking. Proceedings of 23rd IEEE International Conference Image and Vision Computing New Zealand (IVCNZ) (pp. 1-6).

[10] Zhang, L., Wu, X., Buades, A., \& Li, X. (2011). Color demosaicking by local directional interpolation and non-local adaptive thresholding. Journal of Electronic Imaging, 20(2), 023016-023016-16.

[11] Li, J. S. J., \& Randhawa, S. (2009). Color filter array demosaicking using high-order interpolation techniques with a weighted median filter for sharp color edge preservation. IEEE Transactions on Image Processing, 18(9), 1946-1957.

[12] Chen, X., He, L., Jeon, G., \& Jeong, J. (2015). Multidirectional weighted interpolation and refinement method for Bayer pattern CFA demosaicking. IEEE Transactions Circuits Systems Video Technology, 25(8), 1271-1282.

[13] Li, J. S. J., \& Randhawa, S. (2013). Adaptive CFA demosaicking using bilateral filters for colour edge preservation. Proceedings of 2nd IAPR Asian Conference on Pattern Recognition (ACPR) (pp. 451-455).

[14] Wang, L., \& Jeon, G. (2015). Bayer pattern CFA demosaicking based on multi-directional weighted interpolation and guided filter. IEEE Signal Processing Letters, 22(11), 2083-2087.

[15] Li, N., Li, J. S. J., \& Randhawa, S. (2017). Color filter array demosaicking based on the distribution of directional color differences. IEEE Signal Processing Letters, 24(5), 604-608.

[16] Dubois, E. (2005). Frequency-domain methods for demosaicking of Bayer sampled color images. IEEE Signal Processing Letters, 12(12), 847-850.

[17] Leung, B., Jeon, G., \& Dubois, E. (2011). Least-squares luma-chroma demultiplexing algorithm for Bayer demosaicking. IEEE Transactions Image Processing, 20(7), 1885-1894.

[18] Su, C. Y., Lin, C. M., \& Lin, Y. S. (2007). Effective false color suppression of demosaicing using direction inversion and bidirectional signal correlation. Proceedings of 2007 IEEE International Conference on Image Processing: Vol. 2 (pp. 85-88).

[19] Tomaselli, V., Guarnera, M., \& Messina, G. (2009). False colors removal on the ycr-cb color space. Proceedings of IS\&T-SPIE Electronic Imaging Symposium on Digital Photography V (pp. 72500). San Jose, CA, USA. 
[20] Guarnera M., Messina G., \& Tomaselli V. (2010). Adaptive color demosaicing and false color removal. Journal of Electronic Imaging, 19(2), 021105.

[21] Losson, O., Macaire, L., \& Yang, Y. (2010). Chapter 5 - Comparison of color demosaicing methods, survey. Advances in Imaging and Electron Physics. Elsevier, 162, 173-265.

[22] Zhen, R., \& Stevenson, R. L. (2015). Image Demosaicing. Springer International Publishing.

[23] Gunturk, B. K., Glotzbach, J. Altunbasak, Y. Schafer, R. W., \& Mersereau, R. M. (2005). Demosaicking: Color filter array interpolation. IEEE Signal Processing Magazine, 22(1), 44-54.

[24] Omer, I., \& Werman, M. (2004). Color lines: Image specific color representation. Proceedings of the 2004 IEEE Computer Society Conference on Computer Vision and Pattern Recognition (CVPR 2004) (pp. 946-953).

[25] Ono, S., \& Yamada, I. (2016) Color-line regularization for color artifact removal. IEEE Transactions on Computational Imaging, 2(3), 204-217.

[26] Achanta, R., Shaji, A., Smith, K., Lucchi, A., Fua, P., \& Susstrunk, S. (2012). SLIC superpixels compared to state-of-the-art superpixel methods. IEEE Transactions on Pattern Analysis and Machine Intelligence, 34(11), 2274-2282.

[27] Jolliffe, I. (2002). Principal Component Analysis. Springer-Verlag New York.

[28] True color kodak images. Retrieved from http://r0k.us/graphics/kodak

[29] Li, X., Gunturk, B., \& Zhang, L. (2008). Image demosaicing: A systematic survey. Proceedings Volume 6822, Visual Communications and Image Processing 2008.

[30] Chen, X., Jeon, G., \& Jeong, J. (2014). Voting-based directional interpolation method and its application to still color image demosaicking. IEEE Transactions Circuits Systems Video Technology, 24(2), 255-262.

[31] Chung, K., \& Chan, Y. (2006). Color demosaicing using variance of color differences. IEEE Transactions Image Processing, 15(10), 2944-2955.

[32] Xue, W., Zhang, L., Mou, X., \& Bovik, A. C. (2014). Gradient magnitude similarity deviation: A highly efficient perceptual image quality index. IEEE Transactions Image Processing, 23(2), 684-695.

[33] Shakar, O., Li, J. S. J., \& Randhawa, S. (2018). Quantification of color artifacts for the evaluation of color filter array demosaicking. Journal of Image and Graphics, 6(1), 48-53.

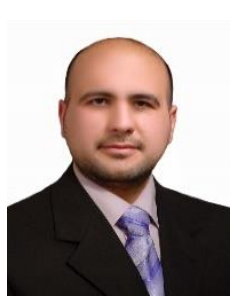

Omar Shakar received the B.E. degree in computer engineering from the University of Technology-Baghdad, in 2004, and M.Eng. degree in computer engineering from the Northern Technical University, in 2012. Currently, he is perusing the Ph.D degree in computer engineering with the College of Science and Engineering at Flinders University in Australia in the area of image processing. His main research interest are color image processing, color filter array demosaicking and pattern recognition.

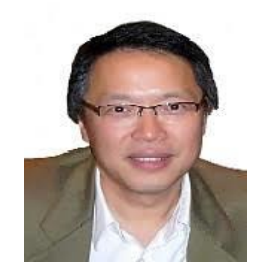

Jim S. Jimmy Li received the B.E. (Hons I) and Ph.D degrees from the University of New South Wales, Australia, in 1985 and 1989, respectively. He is with the College of Science and Engineering at Flinders University in South Australia. He is the head of the video and image processing (VIP) group and research leader of the Centre for Maritime Engineering, Control and Imaging. His research interests include color filter array demosaicking, image enhancement and denoising, image identification, and non-linear image processing.

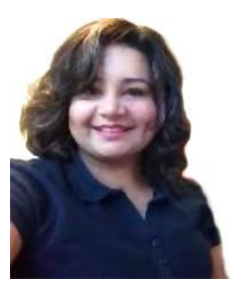

Sharmil Randhawa received the B.E. (Hons) degree from the University of Adelaide, Australia in 1992, and the master of engineering (biomedical engineering) and Ph.D degrees from Flinders University, Australia in 1995 and 2011 respectively. She is with the College of Science and Engineering at Flinders University in South Australia. She is part of 
the video and image processing (VIP) research group in the Centre for Maritime Engineering, Control and Imaging, as well as the Medical Devices and Research Institute (MDRI). She is the vice-chair of the IEEE South Australia section. Her research interests include color filter array demosaicking, denoising, and physiological measurements and monitoring. 